All Nations University Journal of Applied Thought (ANUJAT)

A Multidisciplinary Approach

ALL NATIONS

UNIVERSITY

COLLEGE

Volume 4/ Number 2

May 2015

Article 1

\title{
Analysis of Financial Performance of Chennai Petroleum Corporation Limited, Chennai, India.
}

EMMANUEL ATTAH KUMAH

EMMANUEL ATTAH KUMAH is a PhD Candidate, Research Scholar and Guest Lecturer at Joseph School of Business Studies, Sam Higginbottom University of Agriculture, Technology and Sciences (SHUATS), Allahabad-Utter Pradesh, India.

For this and additional works at:

anujat.anuc.edu.gh

Copyright (C) May 2015 All Nations University Journal of Applied Thought (ANUJAT) and Authors

Recommended Citation:

Attah, E. K. (2015). Analysis of Financial Performance of Chennai Petroleum Corporation Limited, Chennai, India. All Nations University Journal of Applied Thought (ANUJAT), 4(2): 1-18. All Nations University Press.

Available at:

http://anujat.anuc.edu.gh/universityjournal/anujat//ol4/No2/1.pdf

Research Online is the Institutional repository for All Nations University College.

For further information, contact the ANUC Library: anujat@anuc.edu.gh 


\begin{abstract}
Financial analysis of mining projects can be known by studying the financial statements. Financial statements are official records of the financial actions of a company, firm or other unit over a period of time which provide a general idea of a company's financial situation in mutually short and long term. They give a precise representation of a company's condition and working results. Financial statements are sometimes used as supervision tool mainly by company executives and investor's in assessing the overall situation and working results of the company. An effort has been made in this study to analyze the financial conditions of Chennai Petroleum Ltd. This study report on ratio analysis assesses the financial strengths and weakness of Chennai Petroleum Ltd. through Financial Ratio Analysis; to evaluate the performance of the company by using ratios as a yardstick to measure the efficiency of the company, to understand the liquidity, profitability and efficiency positions of the company during the study period by using comparative balance sheet analysis, to evaluate and analyze various facts of the financial performance of the company using multi discriminant working capital analysis such as Y-Score analysis, Z-Score analysis, trend analysis, to make comparisons between the ratios during different periods by using statement of changes in working capital and making projection of the financial performance of the company using trend analysis.
\end{abstract}

Keywords: Financial Performance, Petroleum Corporation, Ratio Analysis, Y-Score and ZScore analysis and trend analysis

\title{
Introduction
}

Financial statements are official records of the financial actions of a company, firm or other unit over a period of time which provide a general idea of a company or person's financial situation in mutually short and long term. Financial statements are used for supervision tool mainly by company executives and investors in assessing the overall status and financial condition of the company. Financial statements are required for owner, shareholder, employee, future investor, income tax department. An investor who is interested to invest his money in a mining company will be keen to know about the economic performance and financial conditions of the company to ensure profitability and better return on investment. Analysis of financial statements helps in formative the liquidity situation, long term solvency, financial feasibility and prosperity of a firm. Financial ratio analysis show whether the firm is performing well or not in past years. Furthermore, comparison of unlike aspect of the entire firms can be done efficiently with this. It helps the traders to make a decision on whether the threat is less or maximum benefit can be earned. Mining industry is capital demanding. For this reason, a lot of capital is needed to invest in it. Before taking decisions on investing in such company, one has to cautiously study its financial status and worth. Limited studies have been carried out by investigators on financial analysis of mining companies. This study envisages to carry out detailed financial analysis of various financial activities in Chennai Petroleum Ltd. and determination of different financial ratios to provide tools for judicious decision making by the investors and management of the mining company. In short, the firms 
itself as well as various interested groups such as managers, shareholders, creditors, tax authorities, and others seek answers to the Following important questions:

1. What is the financial position of the firm at a given point of time?

2. How is the financial performance of the firm over a given period of time?

These questions can be answered with the help of financial analysis of a firm. Financial analysis involves the use of financial statements.

However, financial statements do not reveal all the information related to the financial operations of a firm, but they furnish some extremely useful information, which highlights two important factors profitability and financial soundness. Thus, analysis of financial statements is an important aid to financial performance analysis. Financial performance analysis includes analysis and interpretation of financial statements in such a way that it undertakes full diagnosis of the profitability and financial soundness of the business.

\section{Results and Discussions}

Financial statement analysis is one of the important tools for analyzing the performance of every business (Bernstein, Leopold A. 1984 and Foster, G. 1978). The comparative balance sheet was used to analyze the performance of the business.

Table 1: Analysis of Financial Statement from 2008 to 2009

\begin{tabular}{|l|l|l|l|l|}
\hline PARTICULARS & $\mathbf{2 0 0 8}$ & $\mathbf{2 0 0 9}$ & $\begin{array}{l}\text { CHANG } \\
\text { ABSOLUTE }\end{array}$ \\
\hline Sources of Funds & & & & \multicolumn{1}{|c|}{} \\
\hline 1. Shareholders' Funds & & & & \\
\hline (a) Capital & & & 0 & 0 \\
\hline (b) Reserves and Surplus & 14900.46 & 14900.46 & & -11.98 \\
\hline Total & 331551.84 & 291823.41 & -39728.43 & -11.46 \\
\hline 2. Loan Funds & 346452.3 & 306723.87 & -39728.43 & \\
\hline (a) Secured Loans & & & & \\
\hline (b) Unsecured Loans & & & & -18.59 \\
\hline Total & 51548.26 & 41961.64 & -9586.62 & -41.68 \\
\hline 3. Deferred Tax Liability (net) & 193497.36 & 112828.86 & -80668.5 & -36.83 \\
\hline Total & 245045.62 & 154790.5 & -90255.12 & -31.83 \\
\hline Application of Funds & 60736.14 & 41400.2 & -19335.94 & -22.89 \\
\hline & 652234.06 & 502914.57 & -149319.49 & \\
\hline 1. Fixed Assets & & & & \\
\hline (a) Gross Block & & & & \\
\hline $\begin{array}{l}\text { (b) Less: Depreciation and } \\
\text { Amortization }\end{array}$ & & & & \\
\hline
\end{tabular}




\begin{tabular}{|l|l|l|l|l|}
\hline (c) Net Block & 301179.37 & 285833.08 & -15346.29 & -5.09 \\
\hline Capital goods work in progress & 29598.48 & 71820.77 & 42222.29 & 142.65 \\
\hline 2. Intangible Assets & & & & \\
& & & & \\
\hline (a) Gross Block & 6011.16 & 2563.08 & -3448.08 & -57.36 \\
\hline (b) less: Amortization & 2303.35 & 1212.09 & -1091.26 & -47.37 \\
\hline (c) Net Block & 3707.81 & 1350.99 & -2356.82 & -63.56 \\
\hline Total & 334485.66 & 359004.84 & 24519.18 & 7.33 \\
\hline 3. Investments & 11046.61 & 2281.41 & -8765.2 & -79.34 \\
\hline $\begin{array}{l}\text { 4. Current Assets Loans and } \\
\text { Advances }\end{array}$ & & & & \\
& & & & \\
\hline (a) Inventories & & & & \\
\hline (b) Sundry Debtors & 151069.45 & 101342.28 & -49727.17 & -32.91 \\
\hline (c) Cash and Bank Balances & 1466.5 & 962.69 & -503.81 & -34.35 \\
\hline $\begin{array}{l}\text { (d) Other Current Assets Interest } \\
\text { Accrued on Investments/Bank } \\
\text { Deposits }\end{array}$ & 41.57 & 2.36 & -39.21 & -94.32 \\
\hline $\begin{array}{l}\text { T. Less: Current Liabilities } \\
\text { and Provisions }\end{array}$ & & & & \\
\hline (e) Loans and Advances & 19745.31 & 17561.65 & -2183.66 & -11.05 \\
\hline Total & 615526.2 & 366896.7 & -248629.5 & -40.39 \\
\hline (b) Provisions & & & & \\
\hline Total & & & & \\
\hline 6. Net Current Assets (4-5) & 30670506.08 & 223640.14 & -36865.94 & -14.15 \\
\hline Total & 052234.06 & 502914.57 & -149319.49 & -22.89 \\
\hline
\end{tabular}

\section{Interpretation}

1. There was a decrease in the working capital by Rs. 165073.5 lakhs and decrease in the current assets by $40 \%$. This clearly shows that there has been under utilization of the working capital and consequently the investments for the same year have also gone down by $79 \%$.

2. The years reserve and surplus were reduced by $11 \%$ indicating that the profits have gone down compared to the previous financial year. 
3. Though there was $14 \%$ increase in sales, the total expenditure had sharply risen by 16 $\%$ and resulting in reduced profitability margin to the company.

Table 2: Analysis of Financial Statement from 2009 to 2010

\begin{tabular}{|c|c|c|c|c|}
\hline PARTICULARS & 2009 & 2010 & $\begin{array}{l}\text { ABSOLUTE } \\
\text { CHANGES }\end{array}$ & $\begin{array}{c}\text { CHANGE } \\
\%\end{array}$ \\
\hline \multicolumn{5}{|l|}{ Sources of Funds } \\
\hline \multicolumn{5}{|l|}{ 1. Shareholders' Funds } \\
\hline (a) Capital & 14900.46 & 14900.46 & 0 & 0 \\
\hline (b) Reserves and Surplus & 291823.41 & 331308.11 & 39484.7 & 13.53 \\
\hline Total & 306723.87 & 346208.57 & 39484.7 & 12.87 \\
\hline \multicolumn{5}{|l|}{ 2. Loan Funds } \\
\hline (a) Secured Loans & 41961.64 & 40689.66 & -1271.98 & -3.03 \\
\hline (b) Unsecured Loans & 112828.86 & 367099.23 & 254270.37 & 225.35 \\
\hline Total & 154790.5 & 407788.89 & 252998.39 & 163.44 \\
\hline 3. Deferred Tax Liability (Net) & 41400.2 & 57595.45 & 16195.25 & 39.11 \\
\hline Total & 502914.57 & 811592.91 & 308678.34 & 61.37 \\
\hline \multicolumn{5}{|l|}{ Application of Funds } \\
\hline \multicolumn{5}{|l|}{ 1. Fixed Assets } \\
\hline (a) Gross Block & 516390.1 & 544472.92 & 28082.82 & 5.43 \\
\hline $\begin{array}{l}\text { (b) Less: Depreciation and } \\
\text { Amortization }\end{array}$ & 230557.02 & 253579.43 & 23022.41 & 9.98 \\
\hline (c) Net Block & 285833.08 & 290893.49 & 5060.41 & 1.77 \\
\hline Capital goods work in progress & 71820.77 & 128073.93 & 56253.16 & 78.32 \\
\hline \multicolumn{5}{|l|}{ 2. Intangible Assets } \\
\hline (a) Gross Block & 2563.08 & 3792.33 & 1229.25 & 47.95 \\
\hline (b) Less: Amortization & 1212.09 & 1772.62 & 560.53 & 46.24 \\
\hline (c) Net Block & 1350.99 & 2019.71 & 668.72 & 49.49 \\
\hline Total & 359004.84 & 420987.13 & 61982.29 & 17.26 \\
\hline 3. Investments & 2281.41 & 2342.89 & 61.48 & 2.69 \\
\hline $\begin{array}{l}\text { 4. Current Assets Loans and } \\
\text { Advances }\end{array}$ & & & & \\
\hline
\end{tabular}




\begin{tabular}{|l|l|l|l|l|}
\hline (a) Inventories & 247027.72 & 437824.08 & 190796.36 & 77.23 \\
\hline (b) Sundry Debtors & 101342.28 & 85431.01 & -15911.27 & -15.70 \\
\hline (c) Cash and Bank Balances & 962.69 & 1434.18 & 471.49 & 48.97 \\
\hline $\begin{array}{l}\text { (d) Other Current Assets Interest } \\
\text { Accrued on Investments/Bank } \\
\text { Deposits }\end{array}$ & 2.36 & 1.86 & -0.5 & -21.18 \\
\hline (e) Loans and Advances & & & & \\
\hline Total & 17561.65 & 40633.1 & 23071.45 & 131.37 \\
\hline $\begin{array}{l}\text { 5. Less: Current Liabilities } \\
\text { and Provisions }\end{array}$ & 366896.7 & 565324.23 & 198427.53 & 54.08 \\
\hline (a) Current Liabilities & & & & \\
\hline (b) Provisions & & & & \\
\hline Total & 223640.14 & 154699.17 & -68940.97 & -30.82 \\
\hline 6. Net Current Assets (4-5) & 1628.24 & 22362.17 & 20733.93 & 1273.39 \\
\hline 7. Miscellaneous Expenditure & 0 & 177061.34 & -48207.04 & -21.39 \\
\hline Total & 225268.38 & 0 & 0 & 0 \\
\hline
\end{tabular}

\section{Interpretation}

1. With the expansion of CDU-III and increased capacity utilization of 11.5 MMTPA, the financial year had a great increase in its loans by $163 \%$ and the also the net current assets had risen by $174 \%$.

2. Inventories increased by $77 \%$ and the cash and bank balances increased by $49 \%$ indicating a significant rise in the profitability of the concern.

3. The sales had decreased by $22 \%$ and the working capital had increased by $174 \%$ which indicates an effective utilization of the resources and the profits rose to Rs. 60321.95 lakhs.

Table 3: Analysis of Financial Statement from 2010 to 2011

\begin{tabular}{|l|c|c|c|c|}
\hline PARTICULARS & $\mathbf{2 0 1 0}$ & $\mathbf{2 0 1 1}$ & $\begin{array}{c}\text { ABSOLUTE } \\
\text { CHANGES }\end{array}$ & $\begin{array}{c}\text { CHANGE } \\
\text { \% }\end{array}$ \\
\hline Sources of Funds & & & & \\
\hline 1. Shareholders' Funds & & & & \\
\hline (a) Capital & 14900.46 & 14900.46 & 0.00 & 0 \\
\hline
\end{tabular}




\begin{tabular}{|c|c|c|c|c|}
\hline (b) Reserves and Surplus & 331308.11 & 361692.10 & 30383.99 & 3.74 \\
\hline Total & 346208.57 & 376592.56 & 30383.99 & 3.74 \\
\hline $\begin{array}{l}\text { 2. Loan Funds } \\
\text { (a) Secured Loans }\end{array}$ & 40689.66 & 22535.94 & -18153.72 & -2.23 \\
\hline (b) Unsecured Loans & 367099.23 & 399717.33 & 32618.10 & 4.01 \\
\hline Total & 407788.89 & 422253.27 & 14464.38 & 1.78 \\
\hline 3. Deferred Tax Liability (Net) & 57595.45 & 60447.22 & 2851.77 & 0.35 \\
\hline Total & 811592.91 & 859293.05 & 47700.14 & 5.87 \\
\hline $\begin{array}{l}\text { Application of Funds } \\
\text { 1. Fixed Assets }\end{array}$ & & & & \\
\hline (a) Gross Block & 544472.92 & 624627.3 & 80154.36 & 9.87 \\
\hline $\begin{array}{l}\text { (b) Less: Depreciation and } \\
\text { Amortization } \\
\text { (c) Net Block }\end{array}$ & $\begin{array}{l}253579.43 \\
290893.49\end{array}$ & $\begin{array}{l}282792.4 \\
341834.9\end{array}$ & $\begin{array}{l}29212.93 \\
50941.43\end{array}$ & $\begin{array}{l}3.59 \\
6.27\end{array}$ \\
\hline Capital goods work in progress & 128073.93 & 115590.4 & -12483.52 & -1.53 \\
\hline $\begin{array}{l}\text { 2. Intangible Assets } \\
\text { (a) Gross Block }\end{array}$ & 3792.33 & 4215.42 & 423.09 & 0.05 \\
\hline (b) Less: Amortization & 1772.62 & 2420.03 & 647.41 & 0.079 \\
\hline (c) Net Block & 2019.71 & 1745.39 & -274.32 & -0.03 \\
\hline Total & 420987.13 & 459170.7 & 38183.59 & 4.70 \\
\hline 3. Investments & 2342.89 & 2250.45 & -92.44 & -0.01 \\
\hline $\begin{array}{l}\text { 4. Current Assets Loans and } \\
\text { Advances } \\
\text { (a) Inventories }\end{array}$ & 437824.08 & 511298.27 & 73474.19 & 9.05 \\
\hline (b) Sundry Debtors & 85431.01 & 198398.12 & 112967.11 & 13.91 \\
\hline (c) Cash and Bank Balances & 1434.18 & 1236.53 & -197.65 & -0.02 \\
\hline $\begin{array}{l}\text { (d) Other Current Assets Interest } \\
\text { Accrued on Investments/Bank } \\
\text { Deposits }\end{array}$ & 1.86 & 3.36 & 1.50 & 0.0001 \\
\hline (e) Loans and Advances & 40633.1 & 53341.56 & 12708.46 & 1.56 \\
\hline Total & 565324.23 & 764277.84 & 198953.61 & 24.51 \\
\hline $\begin{array}{l}\text { 5. Less: Current Liabilities } \\
\text { and Provisions } \\
\text { (a) Current Liabilities }\end{array}$ & 154699.17 & 338834.27 & 184135.10 & 22.68 \\
\hline
\end{tabular}

\begin{tabular}{|l|l|l|l|l|}
\hline (b) Provisions & 22362.17 & 27571.69 & 5209.52 & 0.64 \\
\hline Total & 177061.34 & 366405.96 & 189344.62 & 23.32 \\
\hline 6. Net Current Assets (4-5) & 388262.89 & 397871.9 & 9608.99 & 1.18 \\
\hline 7. Miscellaneous Expenditure & 0 & 0 & 0.00 & 0 \\
\hline Total & 811592.91 & 859293.1 & 47700.14 & 5.87 \\
\hline
\end{tabular}




\section{Interpretation}

1. This year's financial status has been moderate wit barely notable increase in net current assets by $1.2 \%$ and the working capital has seen a marginal increase of $2 \%$ compared to the previous year.

2. The net sales have increased by $32 \%$ showing effective capital utilization and increase in inventory by $16 \%$.

3. The reserves and surplus has increased by $9 \%$ compared to the previous year showing a better profitability margin.

\section{Statement of Changes in Working Capital}

Table 4: Statement of Changes in Working Capital from 2008 to 2009

\begin{tabular}{|l|l|l|l|l|}
\hline PARTICULARS & $\mathbf{2 0 0 8}$ & $\mathbf{2 0 0 9}$ & $\begin{array}{l}\text { INCREASE } \\
\text { DECEASE }\end{array}$ & $\begin{array}{l}\text { CHANGE } \\
\%\end{array}$ \\
\hline Current Assets & & & & \\
\hline Inventories & 443203.37 & 247027.72 & -196175.65 & -44.26 \\
\hline Sundry Debtors & 151069.45 & 101342.28 & -49727.17 & -32.91 \\
\hline Cash \& Bank Balance & 1466.5 & 962.69 & -503.81 & -34.35 \\
\hline Loans \& Advances & 41.57 & 2.36 & -39.21 & -94.32 \\
\hline Others & 19745.31 & 17561.65 & -2183.66 & -11.05 \\
\hline Total CA (A) & 615526.2 & 366896.7 & -248629.5 & -40.39 \\
\hline Current Liabilities & & & & \\
\hline Liabilities & 260506.08 & 223640.14 & -36865.94 & -14.15 \\
\hline Provisions & 48318.33 & 1628.24 & -46690.09 & -96.63 \\
\hline Total CL (B) & 308824.41 & 225268.38 & -83556.03 & -27.05 \\
\hline Working Capital (A-B) & 306701.79 & 141628.32 & -165073.47 & -53.82 \\
\hline
\end{tabular}

\section{Interpretation}

The year explains a sharp decrease in the working capital and was contributed by an all-time increase in the current liabilities which decreased by $27 \%$; that is Rs. 83556.03 lakhs. The working capital stood at Rs. 141628.32 lakhs which is less by Rs. 165073.5 lakhs when compared to the previous year. 
Table 5: Statement of Changes in Working Capital from 2009 to 2010

\begin{tabular}{|l|l|l|l|l|}
\hline PARTICULARS & $\mathbf{2 0 0 9}$ & $\mathbf{2 0 1 0}$ & $\begin{array}{l}\text { INCREASE } \\
\text { DECEASE }\end{array}$ & $\begin{array}{l}\text { CHANG } \\
\text { \% }\end{array}$ \\
\hline Current Assets & & & & \\
\hline Inventories & 247028 & 437824.08 & 190796.36 & 77.23 \\
\hline Sundry Debtors & 1013428 & 85431.01 & -15911.27 & -15.70 \\
\hline Cash \& Bank Balance & 962.69 & 1434.18 & 471.49 & 48.97 \\
\hline Loans \& Advances & 2.36 & 1.86 & -0.5 & -21.18 \\
\hline Others & 17561.7 & 40633.1 & 23071.45 & 131.37 \\
\hline Total CA (A) & 366897 & 565324.23 & 198427.53 & 54.08 \\
\hline Current Liabilities & & & & \\
\hline Liabilities & 223640 & 154699.17 & -68940.97 & -30.82 \\
\hline Provisions & 1628.24 & 22362.17 & 20733.93 & 1273.39 \\
\hline Total CL (B) & 225268 & 177061.34 & -48207.04 & -21.39 \\
\hline Working Capital (A-B) & 141628 & 388262.89 & 246634.57 & 174.14 \\
\hline
\end{tabular}

\section{Interpretation}

The installation of CDU-III was backed up by a sharp increase in the overall working capital by a percentage of $74 \%$. The current assets increased by $54 \%$ and the increase in inventories by $77 \%$ explain why the sales and profit increased for the financial year.

Table 6: Statement of Changes in Working Capital from 2010 to 2011

\begin{tabular}{|l|l|l|l|l|}
\hline & & & $\begin{array}{l}\text { INCREASE } \\
\text { DECREASE }\end{array}$ & $\begin{array}{l}\text { CHANGE } \\
\text { \%ARTICULARS }\end{array}$ \\
\hline Current Assets & $\mathbf{2 0 1 0}$ & $\mathbf{2 0 1 1}$ & \\
\hline Inventories & 437824.08 & 511298.27 & 73474.19 & 16.78 \\
\hline Sundry Debtors & 85431.01 & 198398.12 & 112967.11 & 132.23 \\
\hline Cash \& Bank Balance & 1434.18 & 1236.53 & -197.65 & -13.78 \\
\hline Loans \& Advances & 1.86 & 3.36 & 1.5 & 80.64 \\
\hline Others & 40633.1 & 53341.56 & 12708.46 & 31.27 \\
\hline Total CA (A) & 565324.23 & 764277.84 & 198953.61 & 35.19 \\
\hline Current Liabilities & & & & \\
\hline Liabilities & 154699.17 & 338834.27 & 184135.1 & 119.02 \\
\hline Provisions & 22362.17 & 27571.69 & 5209.52 & 23.29 \\
\hline Total CL (B) & 177061.34 & 366405.96 & 189344.62 & 106.93 \\
\hline Working Capital (A-B) & 388262.89 & 397871.88 & 9608.99 & 2.47 \\
\hline
\end{tabular}




\section{Interpretation}

The year explained a good increase in sales but a marginal increase in inventories and working capital highlighting the optimum utilization of the stocks. The working capital had a rise of 3 $\%$ when compared to the previous year with notable increase in current liabilities and sundry debtors.

\section{Multi Discriminant Working Capital Analysis- Y Score Analysis}

Y Score $=14.5166 \mathrm{~V}_{2}+0.0015 \mathrm{~V}_{25}+0.8715 \mathrm{~V}_{31}+0.7914 \mathrm{~V}_{35} \mathrm{~V} 2=$ Cash flow $/$

Total tangible assets

V25 = Current asset / Current Liabilities

V31 $=$ Net Sales $/$ Total tangible assets

V35 = Defensive assets / Total operating expenses

\section{V2}

\begin{tabular}{|c|c|c|c|}
\hline Year & $\begin{array}{c}\text { Net Changes in } \\
\text { Cash Flow }\end{array}$ & $\begin{array}{c}\text { Intangible } \\
\text { Assets }\end{array}$ & V2 \\
\hline 2008 & 324.36 & 334485.66 & 2008 \\
\hline 2009 & 503.81 & 359004.84 & 2009 \\
\hline 2010 & 471.49 & 420987.13 & 2010 \\
\hline 2011 & 197.65 & 459170.72 & 2011 \\
\hline
\end{tabular}

V5

\begin{tabular}{|l|c|c|c|}
\hline Year & CA & C 1 & V25 \\
\hline 2008 & 615526.2 & 308824.41 & 1.99 \\
\hline 2009 & 366896.7 & 225268.38 & 1.62 \\
\hline 2010 & 565324.23 & 177061.34 & 3.19 \\
\hline 2011 & 764277.84 & 366405.96 & 2.08 \\
\hline
\end{tabular}

V31

\begin{tabular}{|l|l|c|c|}
\hline Year & Net Sales & $\begin{array}{l}\text { Total Tangible } \\
\text { Assets }\end{array}$ & V31 \\
\hline 2008 & 2801860.17 & 927752.18 & 3.02 \\
\hline 2009 & 225268.38 & 655011.19 & 4.87 \\
\hline 2010 & 177061.34 & 858560.61 & 2.90 \\
\hline 2011 & 366405.96 & 1108363.21 & 2.98 \\
\hline
\end{tabular}


Y Score $=14.5166 \mathrm{~V}_{2}+0.0015 \mathrm{~V}_{25}+0.8715 \mathrm{~V}_{31}+0.7914 \mathrm{~V}_{35}$

\begin{tabular}{|l|l|}
\hline Year & Y-Score \\
\hline 2008 & 2.64 \\
\hline 2009 & 4.27 \\
\hline 2010 & 2.55 \\
\hline 2011 & 2.61 \\
\hline AVG & 2.95 \\
\hline
\end{tabular}

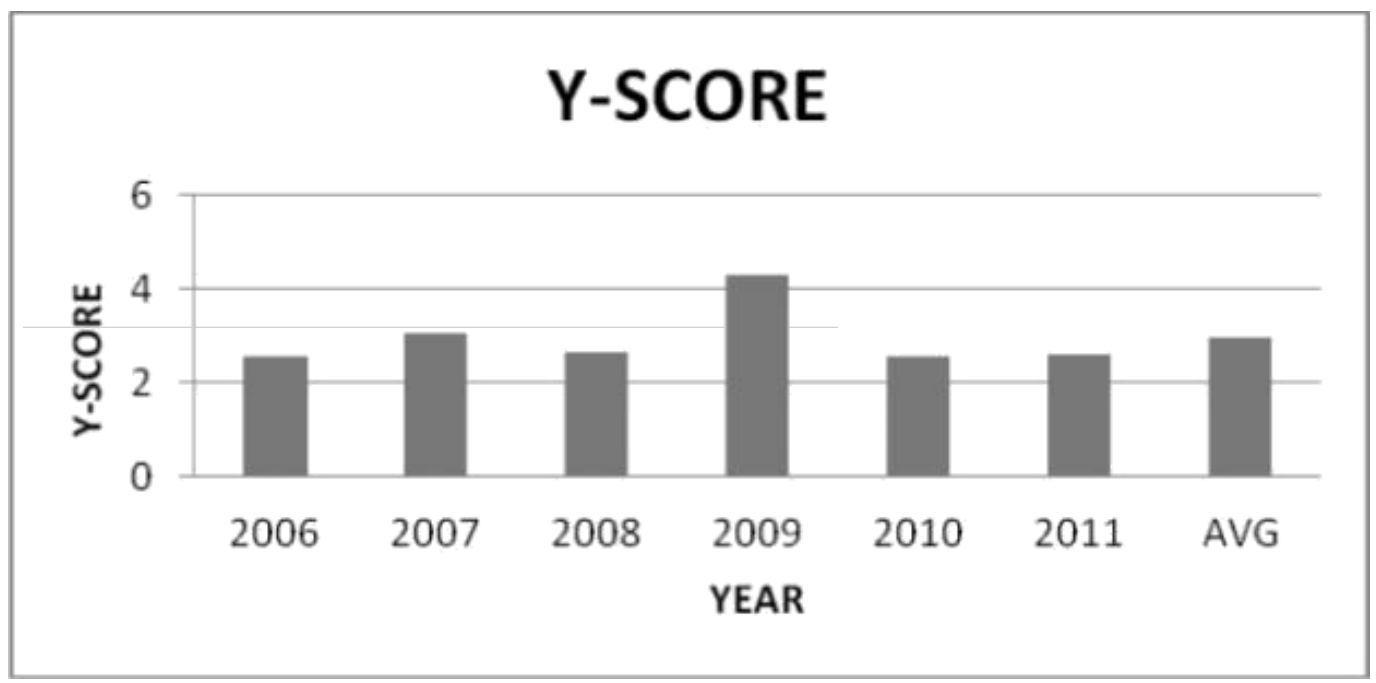

\section{Interpretation}

According to Graham, Benjamin 1975, interpretation of financial statement is very useful for making managerial decision in every organization. To check the overall efficiency of working capital management, the technique of Multi discriminant analysis has been developed, which may also be termed as Y Score analysis. If Y score is above or equal to 1.7069 it is said that management of working capital is effective, otherwise it is ineffective. From the above figures it could be clearly seen that the company is able to manage its working capital effectively. The working capital figures have been pretty consistent over the past few years and also, it's visible that it is rising steadily with increase in net sales.

\section{Z-SCORE}

The $\mathrm{Z}$ score model is a linear analysis in that five measures are objectively weighted and summed up to arrive at an overall score that becomes the basis for classification of firms into one of the priority groupings (distressed and non-distressed). According to Auchterlonie, D.L. 1997, Z score analysis has been done in order to analyze the overall performance evaluation of the company. $\mathrm{Z}$ score is the overall index of corporate health and may be arrived by using the standardized formula. 


$\begin{array}{lll}\mathrm{Z}=1.2 & \mathrm{X} & \text { (Working Capital/Total Assets) } \\ 1.4 & \mathrm{X} & \text { (Retained Earnings/Total Assets) } \\ 0.6 & \mathrm{X} & \text { (Market Value of Equity/Book Value of Debt) } \\ 0.999 & \mathrm{X} & \text { (Sales/Total Assets) } \\ 3.3 & \mathrm{X} & \text { (EBIT/Total Assets) }\end{array}$

Expected Outcome

\begin{tabular}{|l|l|}
\hline Year & Probability of Failure \\
\hline & \\
\hline Less than 1.8 & Very High \\
\hline Greater than 1.81 but less than 2.99 & Not Sure \\
\hline Greater than 3.0 & Unlikely \\
\hline
\end{tabular}

(Working Capital / Total Assets)

\begin{tabular}{|l|l|l|l|l|}
\hline Year & \multicolumn{1}{|c|}{$\begin{array}{c}\text { Total } \\
\text { Assets }\end{array}$} & $\begin{array}{c}\text { Working } \\
\text { Capital }\end{array}$ & & $* 1.2$ \\
\hline 2008 & 927752.18 & 306701.79 & 0.33 & 0.39 \\
\hline
\end{tabular}

\begin{tabular}{|l|l|r|r|r|}
\hline 2009 & 655011.19 & 141628.32 & 0.22 & 0.25 \\
\hline 2010 & 858560.61 & 388262.89 & 0.45 & 0.54 \\
\hline 2011 & 1108363.210 & 397871.88 & 0.36 & 0.43 \\
\hline
\end{tabular}

(Retained Earnings/Total Assets)

\begin{tabular}{|l|l|l|r|r|}
\hline Year & $\begin{array}{c}\text { Retained } \\
\text { Earnings }\end{array}$ & \multicolumn{1}{|c|}{$\begin{array}{c}\text { Total } \\
\text { Assets }\end{array}$} & & $* 1.4$ \\
\hline 2008 & 331551.84 & 927752.18 & 0.35 & 0.50 \\
\hline 2009 & 291823.41 & 655011.19 & 0.44 & 0.62 \\
\hline 2010 & 331308.11 & 858560.61 & 0.38 & 0.54 \\
\hline 2011 & 361692.1 & 1108363.210 & 0.32 & 0.45 \\
\hline
\end{tabular}

(Market Value of Equity/Book Value of Debt)

\begin{tabular}{|l|l|l|l|l|}
\hline Year & \multicolumn{1}{|c|}{ Equity } & \multicolumn{1}{|c|}{ Debt } & & *0.6 \\
\hline 2008 & 346452.3 & 927752.18 & 0.35 & 0.84 \\
\hline 2009 & 306723.87 & 655011.19 & 0.44 & 1.188 \\
\hline 2010 & 346208.57 & 858560.61 & 0.38 & 0.50 \\
\hline 2011 & 376592.56 & 1108363.210 & 0.32 & 0.535118499 \\
\hline
\end{tabular}


(Sales / Total Assets)

\begin{tabular}{|l|l|r|r|r|}
\hline Year & Total Assets & Net Sales & & *0.999 \\
\hline 2008 & 927752.18 & 2801860.17 & 3.02 & 3.01 \\
\hline 2009 & 655011.19 & 3196390.63 & 4.87 & 4.87 \\
\hline 2010 & 858560.61 & 2497262.84 & 2.90 & 2.90 \\
\hline 2011 & 1108363.210 & 3310781.82 & 2.98 & 2.98 \\
\hline
\end{tabular}

(EBIT / Total Assets)

\begin{tabular}{|l|l|l|l|l|}
\hline Year & \multicolumn{1}{|c|}{ EBIT } & Total Assets & & \multicolumn{1}{|c|}{.3 } \\
\hline 2008 & 191643.7 & 927752.18 & 0.20 & 0.68 \\
\hline 2009 & -36944.8 & 655011.19 & -0.05 & -0.18 \\
\hline 2010 & 82111.94 & 858560.61 & 0.09 & 0.31 \\
\hline 2011 & 101797.2 & 1108363.210 & 0.09 & 0.30 \\
\hline
\end{tabular}

\begin{tabular}{|l|l|}
\hline Year & Z-Score \\
\hline 2008 & 5.44 \\
\hline 2009 & 6.76 \\
\hline 2010 & 4.81 \\
\hline 2011 & 4.70 \\
\hline AVG & 5.21 \\
\hline
\end{tabular}

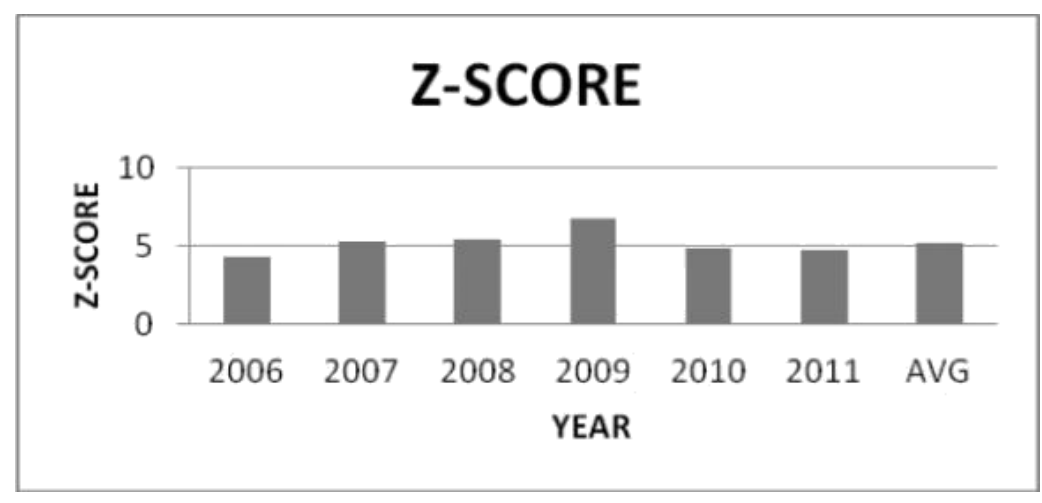

\section{Interpretation}

The result clearly indicates that with an overall score of 5, it is highly impossible for the company to face bankruptcy in the near future or face any sorts of failure that would have a major impact on the company's profit or performance. This explains Altman, E.I. 2000, theory of Predicting Financial Distress of Companies using Z-Score. 


\section{Trend Analysis}

Trend analysis usually involves choosing one fiscal period as a base period and then expressing subsequent quantities as a percentage of the data associated with this base period. The process may involve comparing past and current financial ratios as they related to various institutions in order to project how long the current trend will continue.

\section{Complete Balance Sheet Figures for The Past Six Years}

\begin{tabular}{|l|l|l|l|}
\hline PARTICULARS & $\mathbf{2 0 0 8 0 - 9}$ & $\mathbf{2 0 0 9 - 1 0}$ & $\mathbf{2 0 1 - 1 1}$ \\
\hline Sales & & & \\
\hline PBIT & 3196390.63 & 2497262.84 & 3310781.82 \\
\hline Interest & -36944.82 & 82111.94 & 101797.2 \\
\hline Depreciation & 22366.18 & 13735.53 & 25445.5 \\
\hline PBT & 25716.69 & 26714.19 & 31447.35 \\
\hline PAT & -59311 & 68376.41 & 76351.7 \\
\hline Current Assets & -39728.43 & 60321.95 & 51152.22 \\
\hline Current Liabilities & 366896.7 & 565324.23 & 764277.84 \\
\hline Working Capital & 225268.38 & 177061.34 & 366405.96 \\
\hline Net Fixed Assets & 141628.3 & 388262.89 & 397871.88 \\
\hline Capital Employed & 285833.08 & 290893.49 & 341834.92 \\
\hline Net Worth & 585203.64 & 598371.65 & 857547.66 \\
\hline Debt Equity & 306723.87 & 346208.57 & 376592.56 \\
\hline EPS & 154790.50 & 407788.89 & 422253.27 \\
\hline Dividend (\%) & -26.68 & 40.51 & 34.35 \\
\hline
\end{tabular}

\section{Trend analysis forecasting}

According to Ohlson, J.A. Spring 1980, financial ratios are used to predict future performance of the business. Trend analysis can also be used to forecast the performance of the company in the near future and this is done in two ways:

1. Trend analysis forecasting, and

2. Trend analysis forecasting by extrapolation of the trend lines. 
Trend analysis forecasting

\begin{tabular}{|l|r|l|l|}
\hline Year & Net Sales & Profit & Working Capital \\
\hline 2008 & 2801860.17 & 112295.4 & 306701.79 \\
\hline 2009 & 3196390.63 & 39728.43 & 141628.32 \\
\hline 2010 & 2497262.84 & 60321.95 & 388262.89 \\
\hline 2011 & 3310781.82 & 51152.22 & 397871.88 \\
\hline
\end{tabular}

With the given data for 4 years, the future sales, profit and working capital can be predicted for the next 4 years using excel spreadsheet which uses slope and intercept formulas to forecast the required values. The values are given below:

\begin{tabular}{|l|l|l|l|}
\hline Year & \multicolumn{1}{|c|}{ Net Sales } & \multicolumn{1}{|c|}{ Profit } & Working Capital \\
\hline Slope & 184801.99 & 1311.54 & 39608.06 \\
\hline Intercept & 368443392.4 & 2695596.63 & 79282494.65 \\
\hline
\end{tabular}

Projected Figures

\begin{tabular}{|l|r|c|l|}
\hline Year & Net Sales & Profit & Working Capital \\
\hline 2012 & 3378226.95 & 56763.21 & 408941.61 \\
\hline 2013 & 3563028.95 & 55451.66 & 448549.68 \\
\hline 2014 & 3747830.95 & 54140.12 & 488157.75 \\
\hline 2015 & 3932632.95 & 52828.57 & 527765.82 \\
\hline
\end{tabular}

Trend analysis forecasting using trend line

Trend analysis forecasting can be done by drawing a trend line with reference to the previous years of data. The data for six years are given below for which trend analysis forecasting is to be carried out.

\begin{tabular}{|l|l|l|l|}
\hline Year & Net Sales & Profit & Working Capital \\
\hline 2008 & 2801860 & 112295.4 & 306701.79 \\
\hline 2009 & 31963901 & 39728.43 & 141628.32 \\
\hline 2010 & 2497263 & 60321.95 & 388262.89 \\
\hline 2011 & 3310782 & 51152.22 & 397871.88 \\
\hline
\end{tabular}




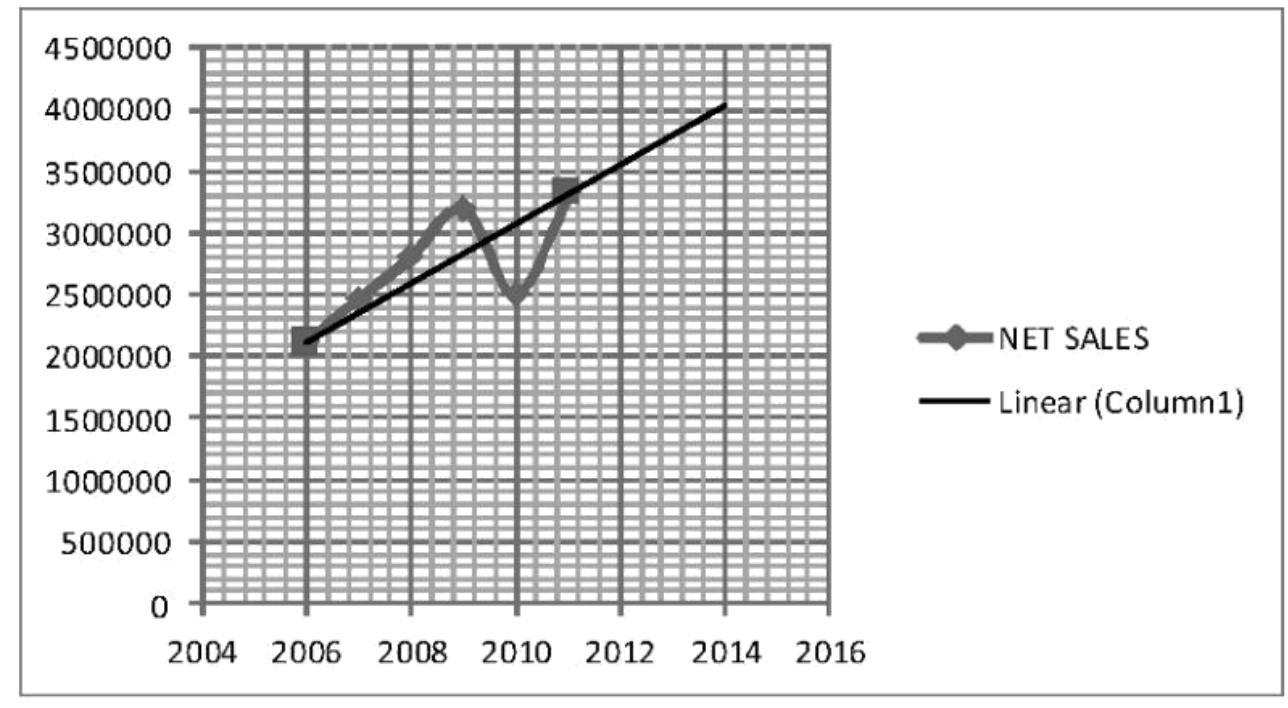

Sales vs net profit

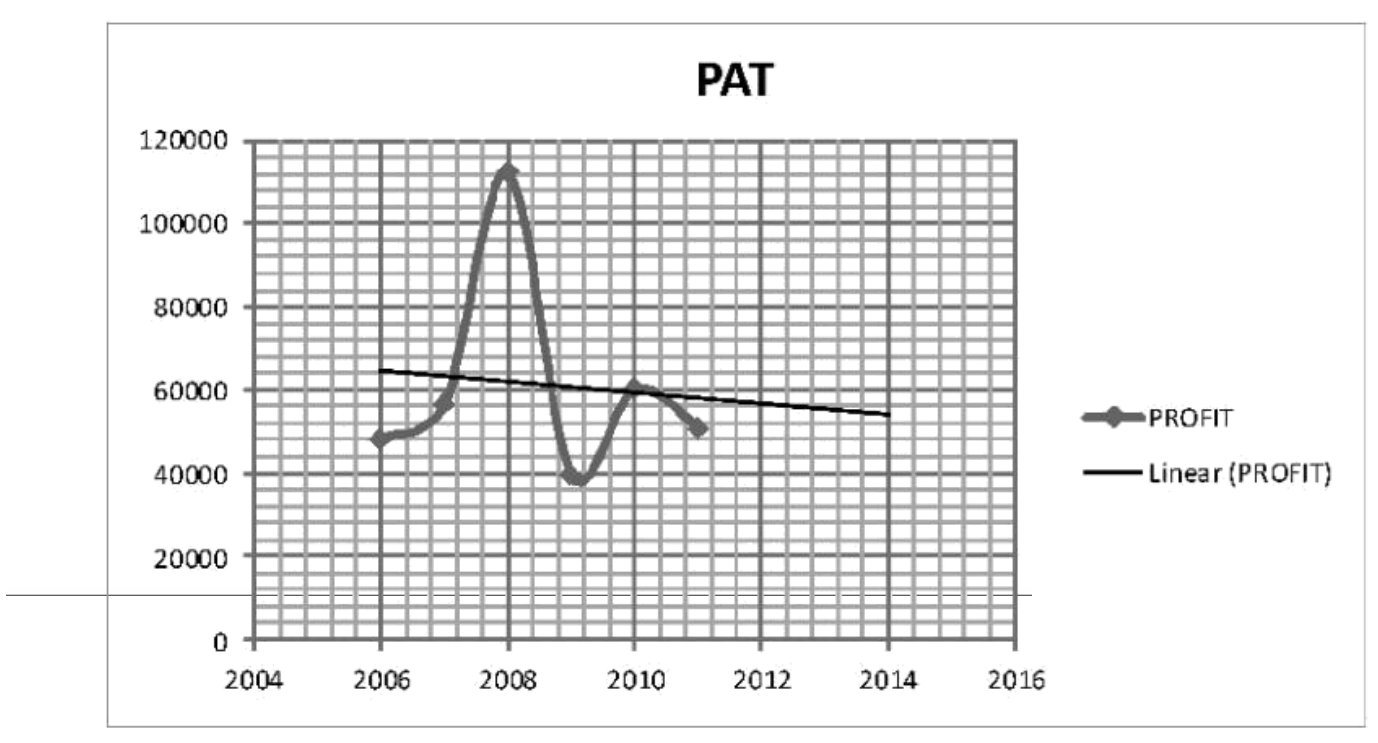

Pat vs Sales

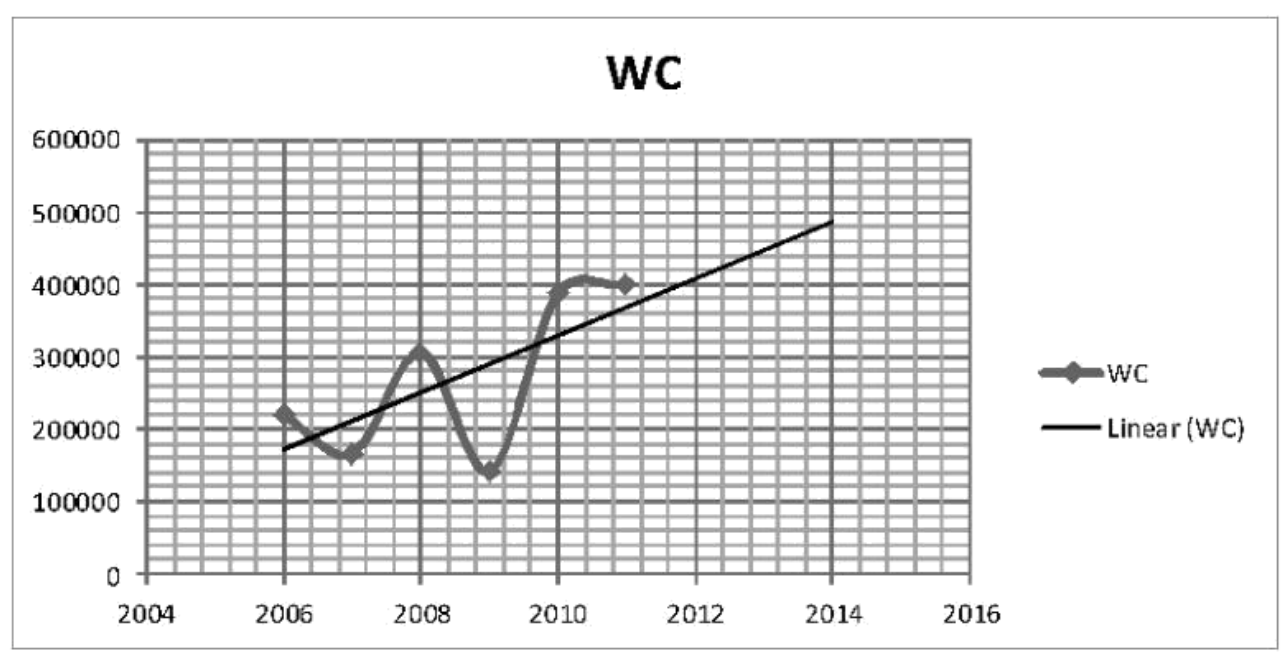

Working capital vs sales 


\section{Projected figures}

\begin{tabular}{|c|c|c|c|}
\hline Year & Net Sales & Profit & Working Capital \\
\hline 2012 & 3500000 & 56000 & 405000 \\
\hline 2013 & 3750000 & 54000 & 450000 \\
\hline 2014 & 4000000 & 52000 & 495000 \\
\hline 2015 & 4250000 & 50000 & 540000 \\
\hline
\end{tabular}

\section{Findings}

1. The two-trend analysis provides us with tentative sales, profit and working capital figures which seem to be approximately similar in both the methodologies providing us with a seemingly accurate data for the future.

2. These data are handy in determining the profitability for the firm but it's highly impossible to deduce accurate information on the profit of the firm as it is interdependent on various other parameters apart from sales, for instance operating expense, interest to be paid and the capital employed. This clearly justifies the concept by Saville, A. in 2004, "Improving the Usefulness of Accounting Data in Financial Analysis."

3. The net sales as been constantly increasing over the past few years and the trend indicates 8-9 \% increase and the trend line has been drawn with this in mind and the sales figures has been estimated. It is observed that at the end of 2015 an estimated sale of above $29 \%$ can be observed assuming the current year to be the base.

4. An annual increase of $10-11 \%$ can be observed in the working capital and an overall increase of 38-39\% for the next 4 years with 2011 as base can be obtained.

5. The profit of the company has been fluctuating and is difficult to determine the exact trend variations; nevertheless, the analysis indicates a definite drop in the profit for about $3 \%$. It is to be noted that the profits of the company is interdependent on various factors and sales is one important parameter based on which the analysis has been formulated.

\section{Conclusion}

The trend analysis results show that the company will show increased sales figure with moderate increase in its profits and strengthened working capital and its effective utilization. The study on the financial performance analysis indicates that the company has been following a steady growth pattern in its profit and its expansion and adequate amount of projects in hand clearly indicates that the company is on par with any of its competitors and will seemingly go ahead to provide a better financial pattern in the years to come. 


\section{Recommendations}

1. The company is effective in managing its costs like miscellaneous expenditure etc but it can further try cost effective techniques like $\mathrm{ABC}$ analysis to save more cost.

2. The loan amount has been increasing over a few years owing to the increasing number of projects in hand and also its expansion of capacity utilization indicating the growth of the company. The loan amount has increased by $6 \%$ and consequently the reserves and surplus have also been risen which is a good sign for the financial health of the company and more utilization from these amounts can be encouraged to strengthen the company's stability. Profit maximization is important in financial management of a company (Gitman, L.J. 2004 and Pandey,I M 2010).

3. There has been a notable decrease in the cash and bank balances which can be checked in as it is also a valuable asset that would be handy in the future. It is a measure of safety by keeping the reserves and surplus and the cash balance of the company growing.

4. The investments have also decreased by an amount of $10 \%$ in the past six years which can be checked.

5. Defensive assets which are low risk returns can be increased and the company can invest more on its $\mathrm{R} \& \mathrm{D}$ policies which can be fruitful in the long run.

6. The debt equity ratio has decreased from 1.20 to 1.12 in the past six years and this is because of rising in borrowings to fund and the rising cost of Raw Material and for the CDU-III expansion 11.5 MMTPA. This also indicates that the lenders have higher claim over net assets and needs to be concentrated. This prove the concepts in the "Return on Equity" by De Wet, J.H.v.H. \& du Toit, E. 2007 which clearly explain that increasing debt affect shareholder's returns. The higher the debt, the lesser the returns. 


\section{References}

Altman, E.I. (2000) Predicting Financial Distress of Companies: Revisiting the Z-Score and Zeta® Models.

Auchterlonie, D.L. (1997) A paean to the Z-score and its commercial bankruptcy prediction. The Journal of Lending \& Credit Risk Management, pp. 33-44.

Beaver, W.H. 1966, Financial Ratios as Predictors of Failure. International Journal of Digital Accounting Research, vol.1, no.1, ppt. 69-85.

Bernstein, Leopold A. (1984) Analysis of Financial Statements. Commercial publishers.

Graham, Benjamin (1975) The Interpretation of Financial Statements.

Harper Business: A Division of Harper Collins Publishers.

De Wet, J.H.v.H. \& du Toit, E. (2007). Return on Equity: A popular, but f 1 a w e d m e a s u re of c orporat efinancialperfor mance. S.Afr.J.Bus.Manage

Foster, G. (1978) Financial Statement Analysis. New Jersey: Prentice-Hall. Gaap Monitoring Panel Charter.

Gitman, L.J. (2004) Principles of Managerial Finance. New York: Addison Wesley Publishing Company.

Ohlson, J.A. (1980) Financial Ratios and the Probabilistic Prediction of Bankruptcy. Journal of Accounting Research, pp.109-131.

Saville, A. (2004) Improving the Usefulness of Accounting Data in Financial Analysis. South African Journal of Economic and Management Sciences, vol.7, no.3, pp. 504-520

Pandey, I M (2010) Financial Management. Noida, Vikas Publishing house Pvt Ltd, 10th edition, pp.2-36 